\title{
VariantSpark, A Random Forest Machine Learning Implementation for Ultra High Dimensional Data
}

\author{
Arash Bayat ${ }^{1, *}$, Piotr Szul ${ }^{2}$, Aidan R. O'Brien ${ }^{1}$, Robert Dunne ${ }^{2}$, Oscar J. \\ Luo $^{1}$, Yatish Jain ${ }^{1}$, Brendan Hosking ${ }^{1}$, Denis C. Bauer ${ }^{1}$ \\ 1 Health and Biosecurity, CSIRO, Sydney, Australia \\ 2 Data61, CSIRO, Sydney, Australia \\ * arash.bayat@csiro.au
}

\section{Abstract}

The demands on machine learning methods to cater for ultra high dimensional datasets, datasets with millions of features, have been increasing in domains like life sciences and the Internet of Things (IoT). While Random Forests are suitable for "wide" datasets, current implementations such as Google's PLANET lack the ability to scale to such dimensions. Recent improvements by Yggdrasil begin to address these limitations but do not extend to Random Forest. This paper introduces CursedForest, a novel Random Forest implementation on top of Apache Spark and part of the VariantSpark platform, which parallelises processing of all nodes over the entire forest. CursedForest is 9 and up to 89 times faster than Google's PLANET and Yggdrasil, respectively, and is the first method capable of scaling to millions of features.

\section{Introduction}

The ongoing digital revolution is causing a dramatic increase in data collected about almost every aspect of life [12]. These datasets are not only growing by capturing more events (samples) but also by capturing more information about these events (features). The challenge of "big" and "wide" data is especially pronounced in the biomedical space where, for example, whole genome sequencing technology enables researchers to extract over 3 billion features from the human genome for analysis [13. Other domains are also 
seeing a rapid increase in the number of features processed by statistical or machine learning applications [1].

While statistical linear models can deal with such wide datasets by analyzing each feature independently [7], there is a growing demand for more realistic approaches that can discover interacting features using machine learning [10]. In the life-science space, this would allow modeling the interactions between genes that result in complex traits like height [9] or diseases like obesity [11]. In particular, Decision Tree [10] based models have been successfully applied to uncover interactions between features [18].

As Decision Trees fitting algorithms are greedy and may yield an estimate with a high variance, Breiman developed an ensemble approach for improving accuracy by aggregating over a large number of Decision Trees, called Random Forest [5]. Random Forest models are particularly well suited for datasets that are wide and there is a need to capture interactions for two reasons:

- Wide datasets, particularly when there are more features than samples, cause other machine learning methods to overfit easily, whereas Random Forest models are more resistant to this "curse of dimensionality" [2, 4]

- There is significant scope for parallelization in Random Forest algorithms allowing forests to be grown efficiently even on large datasets.

The first implementations of Random Forest in $R$ (randomForest) were based on the original Fortran code by Breiman [5]. Later ranger [17] provided a $C++$ implementation of Random Forest with an $R$ interface, which also covers the loading and pre-processing. H2O [8] provides another $R$ interfacing implementation with Scala back end. In terms of parallelization, all of these implementations are optimized for a high performance computing $(H P C)$ machine (a single computing node). However, the Random Forest algorithm allows for parallelization on a distributed computing platform.

Apache Spark is particularly suitable for such massively parallel interconnected calculations as it offers a distributed computing architecture that enables communication beyond compute-node boundaries in a standardized approach [13. In the Spark cluster, there are several Workers and a Master each of which is a computing node in the network. The Driver program (run on the Master node) coordinates the job flow by controlling the Executors (run on the Worker nodes). 
Table 1: Methods evaluated

\begin{tabular}{l|ccc} 
Evaluated Methods & $\begin{array}{c}\text { Implemented } \\
\text { Language }\end{array}$ & $\begin{array}{c}\text { Computational } \\
\text { Platform }\end{array}$ & Reference \\
\hline CursedForest & Scala & Spark Cluster & Our work \\
Google's PLANET (Spark MLlib) & Scala Cluster & Spark & {$[3]$} \\
Yggdrasil & Scala & Spark Cluster & {$[1]$} \\
H2O & Scala \& $R$ & Proprietary Cluster & {$[8]$} \\
randomForest & Fortran \& $R$ & HPC & {$[5]$} \\
ranger & $C++\& R$ & $H P C$ & {$[17]$}
\end{tabular}

Google's PLANET [3] is a MapReduce implementation of Random Forest and the first to parallelize processing each node of a tree in a distributed fashion. Hence, the ideas from Google's PLANET are now used in many "Big Data" machine learning libraries, such as Spark MLlib and XGBoost [6]. Google's PLANET partitions data by samples with Workers holding all features for a fraction of samples. However, this solution produces an approximate split and limits the depth of tree for high dimensional datasets [1].

Yggdrasil [1] overcomes this limitation by flipping the dataset and partitioning it by features rather than samples. The Driver aggregates the best local splits computed on the Executors, identifies the best global split, and updates the Executors accordingly. However, the work is limited to Decision Trees and does not implement bootstrapping or mtry (number of features considered at each node), which are essential components in a Random Forest.

CursedForest extends Yggdrasil's approach to Decision Trees to Random Forest models. CursedForest also introduces a novel method of parallelization in the tree growing process such that nodes of different trees are processed in parallel. This enables highly accurate multivariate models to be built on large datasets with millions of features. For more details see Section 2.3 .

In this paper we evaluated different implementations of Random Forest, including Google's PLANET, for their ability to scale to large datasets (Section 3.1). Section 3.2 and Section 3.3 tests the limits of CursedForest and Section 3.4 benchmarks CursedForest against Yggdrasil. Details of CursedForest are elaborated in Section 2.3 . 
Table 2: Datasets used for evaluations

\begin{tabular}{c|lrr} 
Dataset & \multicolumn{1}{|c}{ Description } & Samples & \multicolumn{1}{c}{ Features } \\
\hline $80 \mathrm{M}$ & 1000 Genomes Phase 3 chr1 to chr22 & 2,540 & $81,047,467$ \\
$20 \mathrm{M}$ & 1000 Genomes Phase 3 chr1 to chr3 & 2,504 & $19,328,051$ \\
$6 \mathrm{M}$ & 1000 Genomes Phase 3 chr1 & 2,504 & $6,450,364$ \\
$1 \mathrm{M}$ & 1000 Genomes Phase 3 chr22 & 2,504 & $1,103,548$ \\
$500 \mathrm{~K}$ & 1000 Genomes Phase 1 chr 22 & 1,092 & 490,036
\end{tabular}

\section{Methods}

\subsection{Datasets}

For the evaluation, well-known genomic datasets (1000 Genomes Project [14]) along with a synthetic dataset are used. The genomic dataset is subsetted to create 5 datasets of different size (see Table 2). Each dataset includes the genomic profile as well as the ethnicity of a few thousand individual humans. In our evaluation, the person's ethnicity (response variable) is predicted from his or her genomic profile and evaluated against the known ethnicity. The genomic profile is a set of features taking the values $0 / 0,0 / 1$ or $1 / 1$ which we encode as 0,1 and 2 respectively.

For the synthetic dataset, we use the method provided by Wright and Ziegler [17]. The synthetic dataset consists of $n$ samples and $p$ features where $p>>n$ and values for each feature are ordinal with three levels represented as numbers 0,1 and 2 (which correspond to an additive effect encoding of genomic variation) randomly generated from a uniform distribution with equal probabilities. For all synthetic dataset, the response variable is a function of five randomly selected features.

The model parameters we use for simulations are $w_{i}=1 / \sqrt{2^{i}-1}$ for $i=1, \cdots, 5$ and we set $z=\sum_{i=1}^{5} w_{i} x_{i}$. We let $\sigma_{\epsilon}^{2}=\operatorname{Var}(z)(1-\theta) / \theta$ where $\theta$ is a parameter controlling the fraction of variance explained by the informative features, and in our study we chose $\theta=0.125$. Then $y=z+\epsilon$ where $\epsilon \sim N\left(0, \sigma_{\epsilon}^{2}\right)$. The dichotomous response is generated by thresholding $y$ at the 0.5 quantile:

$$
\ddot{y}= \begin{cases}0 & \text { for } y \geq Q 2(y) \\ 1 & \text { for } y>Q 2(y)\end{cases}
$$


Table 3: Computational resources used in our experiment

\begin{tabular}{l|lll} 
& Description & $\begin{array}{l}\text { Number of } \\
\text { Executors }\end{array}$ & $\begin{array}{l}\text { Total } \\
\text { Memory } \\
\text { (GB) }\end{array}$ \\
\hline C1 & $\begin{array}{l}\text { A Spark 1.6.1 cluster with 12 worker nodes } \\
\text { each with 16 Intel Xeon E5-2660@2.20GHz } \\
\text { CPU cores and 128 GB of memory }\end{array}$ & $32 \times 8$ \\
\hline C2 & $\begin{array}{l}\text { A Spark 1.6.1 cluster with 12 worker nodes } \\
\text { each with 16 Intel Xeon E5-2660@2.20GHz } \\
\text { CPU cores and 128 GB of memory }\end{array}$ & $130 \times 8$ \\
\hline C3 & $\begin{array}{l}\text { An Amazon aws emr cluster with r4.2xlarge } 8 \times 16 \\
\text { machine as master and8 r4.4xlarge machines } \\
\text { as worker nodes }\end{array}$ & $8 \times 61$ \\
\hline
\end{tabular}

\subsection{Computational resources}

Computational platform described in Table 3 are used in our experiments.

\subsection{CursedForest implementation}

CursedForest implements the original algorithm of Breiman [5]. Given the dataset of $n$ samples and $p$ features, in our implementation, the dataset is partitioned by features and each partition is allocation in an Worker such that the $i^{\text {th }}$ Worker holds $p_{i}$ features for all samples where $\sum_{i} p_{i}=p$. To build each tree, the Driver first bootstrap samples thus different bootstrapped set of samples are used to build each tree. Then, for each node of each tree mtry different features are randomly picked from the dataset to compute Gini Impurity (parallelized over all Executors). Since features are partitioned across Workers, Each Executor (assume one per Worker) randomly pick $m t r y \times p_{i}$ features and finds the best split locally for the node. The Driver aggregates all local best split for each node finds the global best split and updates all Executors about the global best split. This process is carried out across $r b s$ trees in parallel where rbs is set by the user. Hence multiple nodes of multiple trees are processed in parallel.

We keep track of the change in Gini Impurity scores after splitting at each node in each tree. This information is used to calculate the Importance Score which is used as a metric to quantify the contribution of each feature 
in classifying the samples.

This implementation avoids communication bottlenecks between the Driver and the Executors as information exchange is minimal allowing it to build large numbers of trees efficiently. Furthermore, CursedForest has memory efficient representation of genomics data, optimized communication patterns, and computation batching. It also provides an efficient implementation of Out-Of-Bag (OOB) error calculation, which substantially simplifies parameter tuning over the more computationally expensive alternative of crossvalidation.

CursedForest is available as a Github repository

The GitHub repository (https://github.com/aehrc/VariantSpark) holds information for setting up on any local or cloud-based computing environment supporting Apache Spark, such as Amazon Web Services and Google Cloud Platform. In addition, CursedForest can also be accessed through a notebook interface hosted at Databricks

(https://aehrc.github.io/VariantSpark/notebook-examples/Variantspark_ HipsterIndex.html). Note that, in addition to $V C F$ and $C S V$ file format, CursedForest also works with the ADAM16 data schema, implemented on top of Avro and Parquet, as well as the HAIL API (https://github.com/ hail-is/hail) for variant pre-processing.

\subsection{Usage of the Gini Impurity score as a data split- ting criterion}

The current implementation of CursedForest uses a Gini Impurity criterion to choose the feature for splitting at each node. This was introduced for Decision Tree in Breiman et al. [10]. For each node $A$ in each tree, except leaf nodes, the program finds the feature (between randomly selected mtry features) that best splits samples in $A$ into two child node $L$ and $R$. Assume node $A$ includes $m$ samples each of which labelled with $q$, where $q \in 1, \ldots, Q$. Given $f_{q}$ as the fraction of samples in the $R$ which are labeled as $q$, then the Gini Impurity of the node $A\left(G i n i_{R}\right)$ is computed as,

$$
\operatorname{Gini}_{R}=1-\sum_{q=1}^{Q} f_{q}{ }^{2} .
$$

Assume $l$ and $r$ are the fractions of samples in $A$ which are moved to $L$ and $R$ respectively $(l+r=1)$ after splitting node $A$ with feature $t$. 
The Information Gained by feature $t\left(I G_{t}\right)$ at node $A$ is computed as Gini Impurity of $A$ minus weighted average of Gini Impurity of $L$ and $R$ using equation,

$$
I G_{t}=\operatorname{Gini}_{A}-\left(x \times G i n i_{L}+y \times G i n i R\right)
$$

The feature that results in the highest Information Gained is selected as the local best split for the node by Executor. When the Driver collects all local best splits it looks for the one that maximizes Information Gained and chooses it as the best global split.

Feature $t$ might be selected as the best split for multiple nodes of multiple trees. The raw Importance Score of feature $t$ is defined as the mean of $I G_{t}$ across all node in the Random Forest where $t$ is chosen as best split.

\subsection{Out-of-Bag training and testing procedure}

The Random Forest algorithm builds each tree on a subset of individuals (approximately two thirds), leaving the other third out. The algorithm then tests each tree against the held-out samples, giving an estimate of the error for each tree. Averaging the error for each tree returns the OOB error for the model. According to Breiman [10, the out-of-bag estimate is as accurate as using a test set of the same size as the training set.

\section{Results and discussion}

\subsection{CursedForest outperforms existing methods for multi- class classification}

Figure 1a shows the execution time of all methods on different sized datasets. Note that not all programs were able to process all datasets due to out of memory errors and time-out. CursedForest is faster than all other methods for all dataset except for the smallest dataset $(500 \mathrm{~K})$ where ranger $R$ implementation is faster (70 seconds vs. 110 seconds). The ranger $R$ is the second fastest implementation of Random Forest but cannot process the largest dataset (80M features). CursedForest is 4.5, 5.3 and 4.2 times faster than ranger $R$ processing $1 \mathrm{M}, 6 \mathrm{M}$, and $20 \mathrm{M}$ dataset respectively. Google's PLANET is the only other Spark implementation of Random Forest but cannot process dataset larger than $6 \mathrm{M}$ features. CursedForest is 9.3 and 


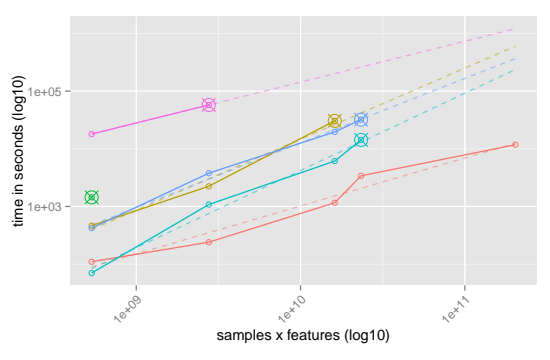

(a) Time consumption

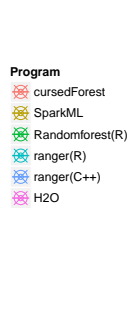

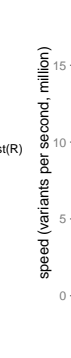

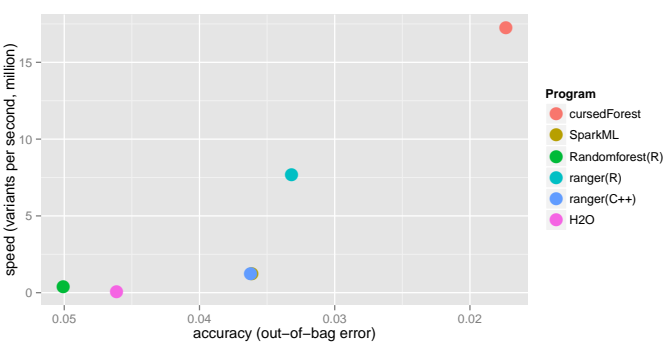

(b) Accuracy vs speed

Figure 1: Comparison of speed and accuracy of CursedForest with other published methods. SparkML refers to Google's PLANET. Crossed cicles $(\otimes)$ mark the last successfully process dataset by the respective methods.

26.4 times faster than Google's PLANET processing 1M and 6M dataset respectively.

CursedForest is the only method able to scale to the largest dataset $(80 \mathrm{M}$ features). This demonstrates that although Google's PLANET was designed to handle many samples, it is unable to efficiently cope with a large number of features. Extrapolating from this, the dotted line in Figure 1a shows that all current implementations would require between 27 hours and 11 days to finish analyzing the largest dataset $(80 \mathrm{M})$, while CursedForest completes the task in about 3 hours on a small cluster set-up (C1).

Figure $1 \mathrm{~b}$ compares accuracy and speed of the different Random Forest implementations on the largest dataset they successfully complete. The accuracy is measured in terms of out-of-bag error rate (lower is better) and the speed is computed as million variants per second (higher is better). CursedForest is the fastest implementation and delivers the most accurate result as it was the only method able to utilize the whole genome (80M).

The runtime of CursedForest can be substantially improved due to its ability to efficiently utilize large numbers of commodity computers. Table 4 shows the runtime when utilizing a large Spark cluster (C2) (see Section 2.2) to run the same analysis as above. We can observe that the speed-up improvement grow with data set size, with up to 5-fold speed-up for 80M (from 11,760 to 2,214 seconds). Also, noteworthy is the reduction in error when the whole data set is utilized. 
Table 4: Accuracy and scalability of a multi-class classification with 50 trees using CursedForest

\begin{tabular}{c|rrrr} 
Dataset & $\begin{array}{c}\text { Error Rate } \\
\text { (OOB) }\end{array}$ & \multicolumn{1}{c}{ C2 } & \multicolumn{1}{c}{ C1 } & Speed up \\
\hline $1 \mathrm{M}$ & 0.06 & 1.6 & 4.0 & 2.5 \\
$6 \mathrm{M}$ & 0.04 & 4.2 & 19.3 & 4.6 \\
$20 \mathrm{M}$ & 0.02 & 10.8 & 56.7 & 5.2 \\
$80 \mathrm{M}$ & 0.01 & 36.9 & 196.0 & 5.3
\end{tabular}

\subsection{CursedForest is linearly scalable with samples, fea- tures and CPUs}

Here, we explore the performance of CursedForest in more detail by testing its ability to scale beyond the real dataset size. We hence generate synthetic datasets with up to 50 million features (i.e., $p=50,000,000)$, with up to 10,000 samples (see Section 2.1).

We measured the runtime to build a binary Random Forest model of 100 trees with a fixed mtry fraction of 0.25 using our C2 computer (see Section 2.2 with these different synthetic datasets. CursedForest scales linearly with increase in feature sizes $p$ and sublinearly with the increase in sample size $n$ (see Figure 2a). CursedForest also scales well when increasing the number of CPUs making cloud-application with on-demand cluster sizes possible (see Figure 2b). As shown in Table 4 the $80 \mathrm{M}$ dataset can be processed in about 37 minutes. With increase in the number of executors the execution time can further decreases.

\subsection{Wide data and the choice of mtry and ntree}

CursedForest is a partial implementation of the original algorithm of [5]. As such the choice of ntree and mtry follows the same logic. However, the original choices of the default values of these parameters was based on Breiman's experience with a number of data sets [5]. CursedForest may be operating in regions where a different set of heuristics may be needed to guide the parameter settings.

In this section, we test the limits of CursedForest in association and classification analysis on high-dimensional data. For this purpose, we generated a synthetic dataset with 2.5 million features (i.e., $p=2,500,000$ ), of which 5 


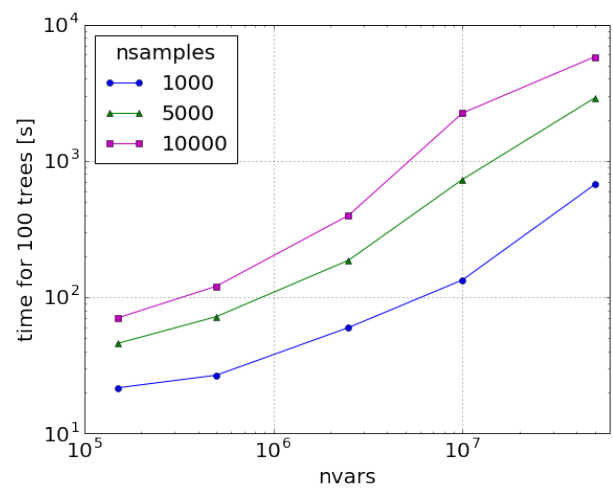

(a) Runtime as a function of increasing cores number of samples and/or features

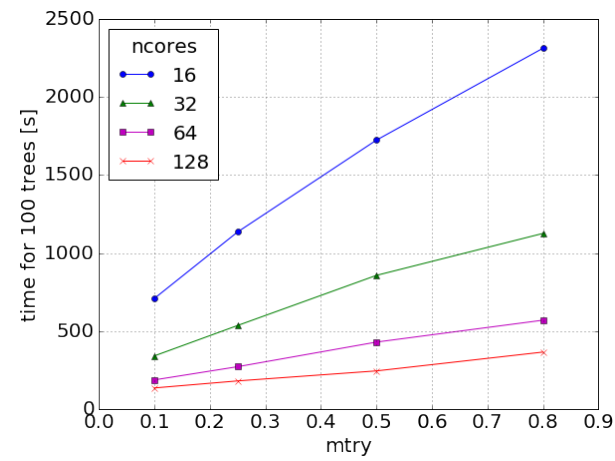

(b) Scalability with increasing number of

Figure 2: Performance in response to varying ntree and mtry

are designed to be related to the response variable, with 5,000 samples (i.e. $n=5,000$ ) (see Section 2.1). We use our C2 computer (see Section 2.2) for this analysis.

We fit the Random Forest model and estimate the classification accuracy by capturing the OOB error. We also measure the feature selection performance by capturing the rank-biased overlap (RBO) measure [16]. RBO assesses whether CursedForest is able to retrieve the 5 features in order of their association weight as a scale from 0 (no feature recovered) to 1 (fully recovered).

See Figure $3 \mathrm{a}$ for plots of these two measures for this example. Note that, for the parameter mtry, the plot shows the proportion, mtry/p, which means that the default value of 1581 is shown as $1581 / p \approx 0.0006$. The default value for mtry does not result in a good classification performance for this large feature dataset. The OOB for this value of mtry does not drop below 0.5, even when the number of trees is increased. Increasing mtry in combination with ntree yields the best performance with the OOB error essentially constant around 0.4 across a large range of mtry and ntree values. This is in contrast to the feature-selection performance, where the RBO measure heavily depends on ntree and gives better results with lower values of mtry (Figure 3b).

This may be because a large mtry leads to more correlated trees as the same important features have a higher chance of being selected in all trees, which does not yield good performance outcomes. This issue is less pro- 


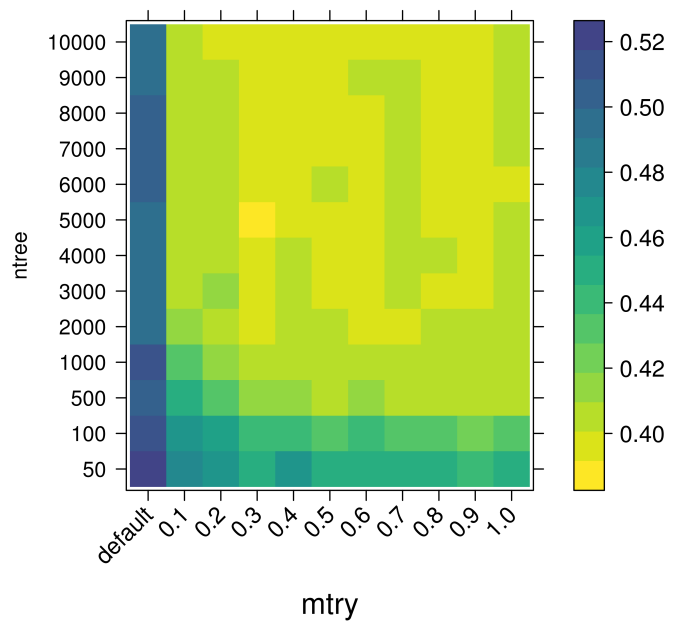

(a) OOB classification error

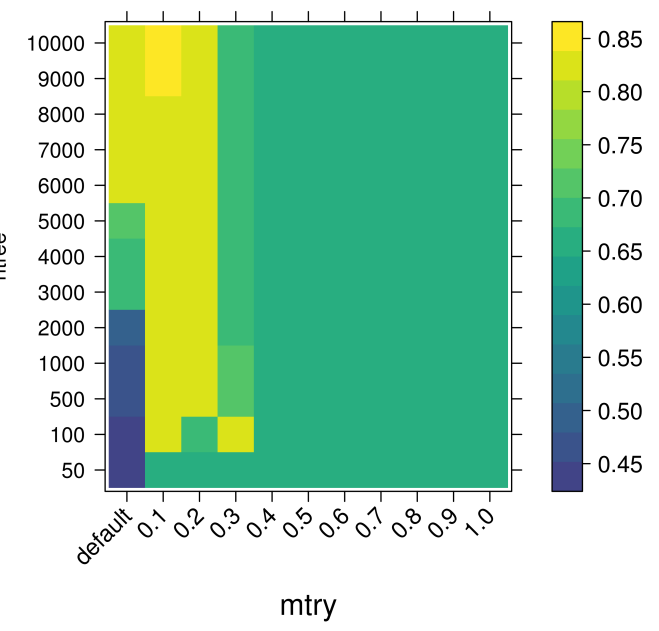

(b) RBO feature selection error

Figure 3: Accuracy performance in response to varying ntree and mtry. mtry is given as a fraction of $p$.

nounced for classification error where random features can mimic the response variable, hence resulting more in a performance plateau. Increasing the number of trees, on the other hand, improves performance especially when the trees are kept diverse (small mtry) but appropriate for large feature datasets (mtry larger than default). The ability of CursedForest to build decision trees in parallel at the compute node level hence caters perfectly to the requirement of large feature datasets as more trees can be built given a fixed time budget.

\subsection{CursedForest tree-building method is faster than Yggdrasil}

Here we compare the tree building implementation of CursedForest to $Y g$ gdrasil. Both methods partition the data by features to parallelize Gini computation, which was demonstrated to yield a better performance for wide data than Google's PLANET, which partitions by samples [1]. Since CursedForest is an ensemble method, we approximate the single Decision Tree functionality of Yggdrasil by (1) disabling bootstrapping, (2) setting $m$ try $=p$, (3) setting $n$ Tree $=1$. 
The largest dataset we were able to process with Yggdrasil is dataset 1M. It took 102 seconds (average over two runs) for Yggdrasil to build a Decision Tree for this dataset, compared to 3 seconds for CursedForest (average over two trees, $m$ try $=p$ and without bootstrapping). This shows nearly 33 times speed up over Yggdrasil. Enabling parallel tree-building reduces the runtime for CursedForest to an average of 1.14 second to build each tree, which represents a 89-fold speed-up over the Yggdrasil implementation. The time mentioned above only include the actual training process, not data loading and other auxiliary steps. For this experiment we use C3 cluster as described in Table 3 .

\section{Conclusion}

The challenge of "big" and "wide" data is especially pronounced in the biomedical space where dataset acquisition is predicted to far outpace that of traditional "Big Data" disciplines [15]. Catering for this, we extended Random Forest to cope with extremely high dimensional data using a novel parallelization approach enabled by Spark. Compared to Google's PLANET and other non-Spark implementations, as well as the purpose-designed $Y g$ gdrasil, CursedForest can scale to millions of features. It also offers the fastest trainings method for Random Forest on a wide range data-sets sizes compared to the other tools tested.

\section{References}

[1] F. Abuzaid, J. K. Bradley, F. T. Liang, A. Feng, L. Yang, M. Zaharia, and A. S. Talwalkar. Yggdrasil: An Optimized System for Training Deep Decision Trees at Scale, volume 29, pages 3817-3825. Curran Associates, Inc., 2016.

[2] D. C. Bauer, C. Gaff, M. E. Dinger, M. Caramins, F. A. Buske, M. Fenech, D. Hansen, and L. Cobiac. Genomics and personalised wholeof-life healthcare. Trends in Molecular Medicine, 20(9):479-486, 2014.

[3] B. P. Bayardo, J. S. Herbach, S. Basu, and R. J. Planet: Massively parallel learning of tree ensembles with mapreduce. In Proceedings of 
the 35th International Conference on Very Large Data Bases (VLDB2009), 2009.

[4] R. Bellman and R. Bellman. Adaptive Control Processes: A Guided Tour. Princeton University Press, 1961.

[5] L. Breiman. Random forests. Machine Learning, 45(1):5-32, 2001.

[6] T. Chen and C. Guestrin. Xgboost: A scalable tree boosting system. In Proceedings of the 22nd acm sigkdd international conference on knowledge discovery and data mining, pages 785-794. ACM, 2016.

[7] W. T. C. C. Consortium. Genome-wide association study of 14,000 cases of seven common diseases and 3,000 shared controls. Nature, 447(7145):661-678, 2007.

[8] H2O. Open-source machine learning platform for enterprises, https://www.h2o.ai/h2o/.

[9] L. Lello, S. G. Avery, L. Tellier, A. Vazquez, G. d. l. Campos, and S. D. Hsu. Accurate genomic prediction of human height. arXiv preprint arXiv:1709.06489, 2017.

[10] C. J. S. R. O. Leo Breiman, Jerome Friedman. Classification and Regression Trees. Wadsworth Publishing Company, Belmont, California, U.S.A., 1 edition, 1984.

[11] A. E. Locke, B. Kahali, S. I. Berndt, A. E. Justice, T. H. Pers, et al. Genetic studies of body mass index yield new insights for obesity biology. Nature, 518(7538):197-206, 2015.

[12] C. Loebbecke and A. Picot. Reflections on societal and business model transformation arising from digitization and big data analytics: A research agenda. The Journal of Strategic Information Systems, 24(3):149157, 2015.

[13] A. R. O'Brien, N. F. W. Saunders, Y. Guo, F. A. Buske, R. J. Scott, and D. C. Bauer. Variantspark: population scale clustering of genotype information. BMC Genomics, 16(1), 2015.

[14] N. Siva. 1000 genomes project, 2008. 
[15] Z. D. Stephens, S. Y. Lee, F. Faghri, R. H. Campbell, C. Zhai, M. J. Efron, R. Iyer, M. C. Schatz, S. Sinha, and G. E. Robinson. Big data: Astronomical or genomical? PLoS Biol, 13(7):e1002195, 2015.

[16] W. Webber, A. Moffat, and J. Zobel. A similarity measure for indefinite rankings. ACM Transactions on Information Systems, 28(4):20:1-20:38, 2010 .

[17] M. N. Wright and A. Ziegler. Ranger: A fast implementation of random forests for high dimensional data in $\mathrm{c}++$ and r. Journal of Statistical Software, 2016.

[18] M. N. Wright, A. Ziegler, and I. R. König. Do little interactions get lost in dark random forests? BMC Bioinformatics, 17(1):145, 2016. 